\title{
Adrenal Schwannoma Treated with Open Adrenalectomy: a Case Report
}

\author{
Prakash Hebbulse Shivalingaiah ${ }^{1,2} \cdot$ Pradeep Kumar $^{3} \cdot$ Suyash Bajoria $^{3}$
}

Received: 24 July 2017 / Accepted: 30 October 2017 /Published online: 9 November 2017

(C) Indian Association of Surgical Oncology 2017

\begin{abstract}
Adrenal schwannomas are very rare tumors accounting for only $0.2 \%$ of the adrenal tumors. These are very difficult to diagnose preoperatively and usually present as incidental non-secreting adrenal masses in asymptomatic patients or in patients with non-specific complaints. Computed tomography (CT) and magnetic resonance imaging (MRI) are required to aid the diagnosis, but adrenal schwannomas cannot be distinguishably confirmed on the basis of imaging alone. Histopathological examination (HPE) is essential for accurate diagnosis. We report a rare case of a right adrenal schwannoma in a 68year-old man. The clinical evaluation was unremarkable and the radiological examination revealed a suprarenal mass lesion on ultrasonography (USG). CT revealed a right adrenal mass which was purported to be likely malignant. Right adrenalectomy was performed by the open approach along with excision of the surrounding enlarged lymph nodes. The postoperative course was uneventful. HPE
\end{abstract}

Prakash Hebbulse Shivalingaiah

drhsprakash@gmail.com

Pradeep Kumar

drpradip.hd@gmail.com

Suyash Bajoria

suyashbajoria89@gmail.com

1 Department of Urology, K. R. Hospital, Mysore, Karnataka, India

2 Mysore Medical College and Research Institute, Mysore, Karnataka, India

3 Department of General Surgery, Mysore Medical College and Research Institute, Mysore, Karnataka, India established the adrenal mass to be a schwannoma, a benign tumor arising from Schwann cells, an exceedingly unusual occurrence in the adrenal glands. A non-secreting adrenal mass can be easily misjudged and the exact diagnosis of the lesion cannot be made sure of without both radiologic and pathologic confirmation. Unilateral adrenal primary or metastatic lesions need pathological confirmation as it can dramatically affect prognosis. Unusual tumors of the adrenal gland like schwannomas may be found incidentally or otherwise and will generate difficulties in establishing the right management. Complete excision is the treatment of choice whenever feasible and will also clarify pathology.

Keywords Incidentaloma $\cdot$ Schwannoma $\cdot$ Adrenal tumor

\section{Introduction}

Schwannomas are benign, slow-growing encapsulated nerve sheath tumors composed of cells with the immunophenotype of Schwann cells. They arise from Schwann cells of peripheral, motor, sympathetic or cranial nerves. They are most commonly seen in head and neck region and upper and lower extremities. Schwannoma may be found in the retro peritoneum and juxta-adrenal area. Schwannomas presenting in visceral organs, especially the adrenal glands are rare.

The adrenal medulla is innervated by the phrenic nerve, the vagus nerve and the sympathetic trunk. Adrenal schwannoma arises from Schwann cells associated with these nerves $[1,2]$. They are predominantly found in females in the second and fifth decades of life [3]. We report a case of adrenal schwannoma discovered incidentally while working up for a case of dysuria. 
Fig. 1 Abdomen CT scan shows well-defined mass lesion in suprarenal area
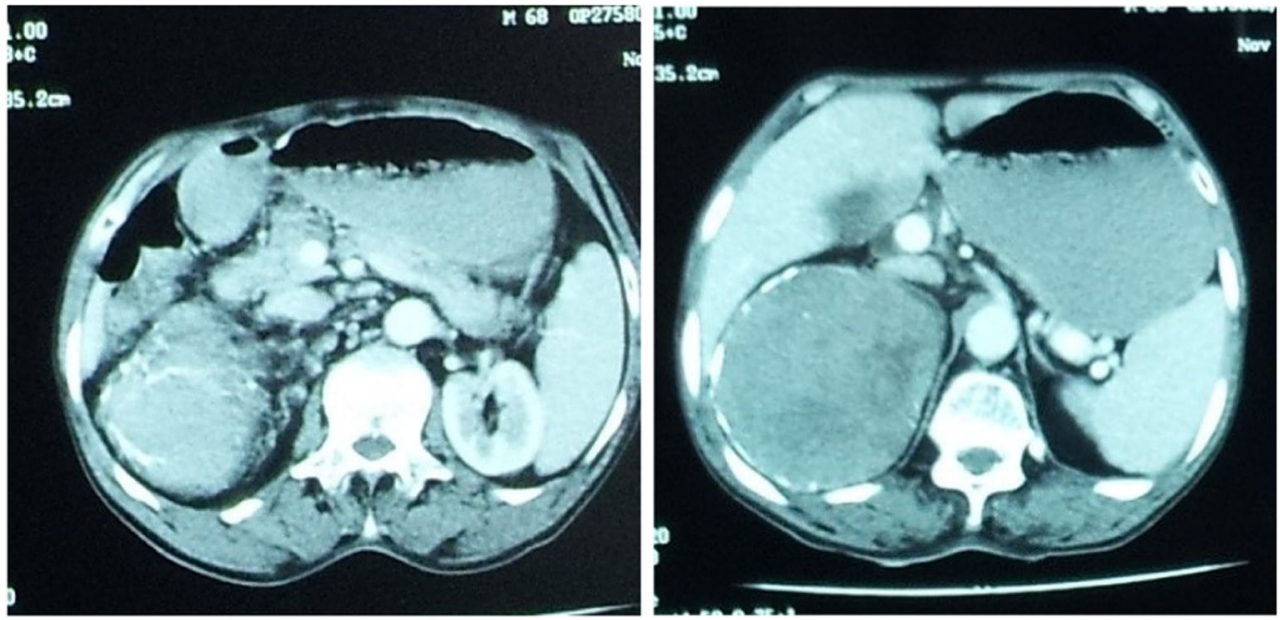

Fig. 2 Grass photograph of adrenal tumor-cross-section shows yellowish white color
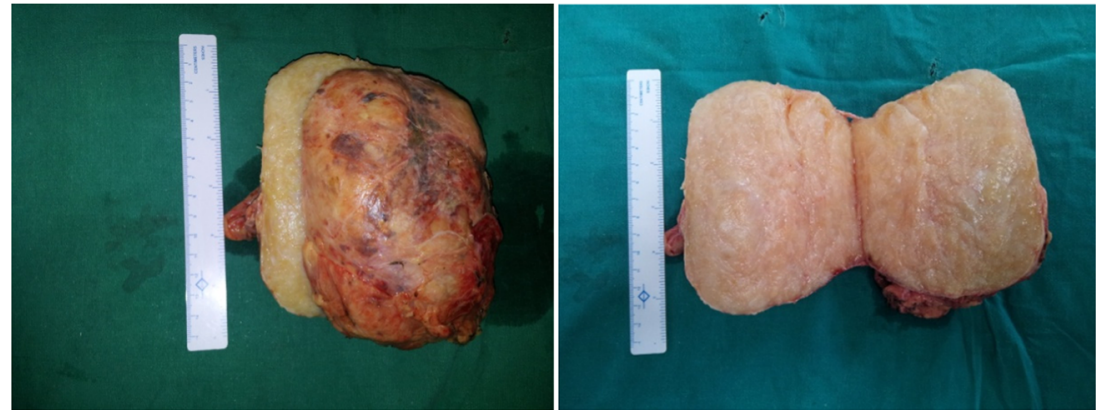

\section{Case Report}

A 68-year-old male patient had difficulty in micturition for 6 months. During evaluation his ultrasonography (USG) abdomen showed a well-defined heterogeneous mass lesion measuring $12 \times 8.5 \mathrm{~cm}$ in the right suprarenal region. Computed tomography of the abdomen and pelvis revealed a well-defined, round, soft tissue density lesion $(40 \mathrm{HU})$ with thin rim of calcification measuring $10.3 \times 10.1 \times 9.7 \mathrm{~cm}$ which is in right suprarenal region (Fig. 1). Right adrenal gland was not separately visualized. On postcontrast study, in venous phase, the lesion showed mild enhancement (60-70 HU), and delayed phase (3 min) showed good enhancement (80-90 HU) with no evidence of immediate wash out.
Metabolic work-ups, including serum electrolytes, cortisol, urinary metanephrine, and VMA, were within normal range.

Due to large size of the tumor, after informed consent, a standard open transperitoneal adrenalectomy was performed. Postoperative period was uneventful. Postoperative pathological evaluation revealed a $380 \mathrm{~g}$ adrenal gland with a solitary tumor measuring $14 \times 8 \times 5 \mathrm{~cm}$ in overall dimensions that was grossly impinging on adrenal parenchyma (Fig. 2).

Histologically, the sections from tumor shows proliferation of spindle-shaped cells arranged in fascicles with intervening hypocellular areas. The cellular area shows long spindle basophilic nucleus with pointing nucleus and poorly defined eosinophilic cytoplasm. Hypocellular areas show nuclei suspended in microcystic matrix (Fig. 3). Immunohistochemical analysis revealed cells that were
Fig. 3 Histologically tumor was composed of spindle-shaped cells arranged in fascicles with intervening hypocellular areas
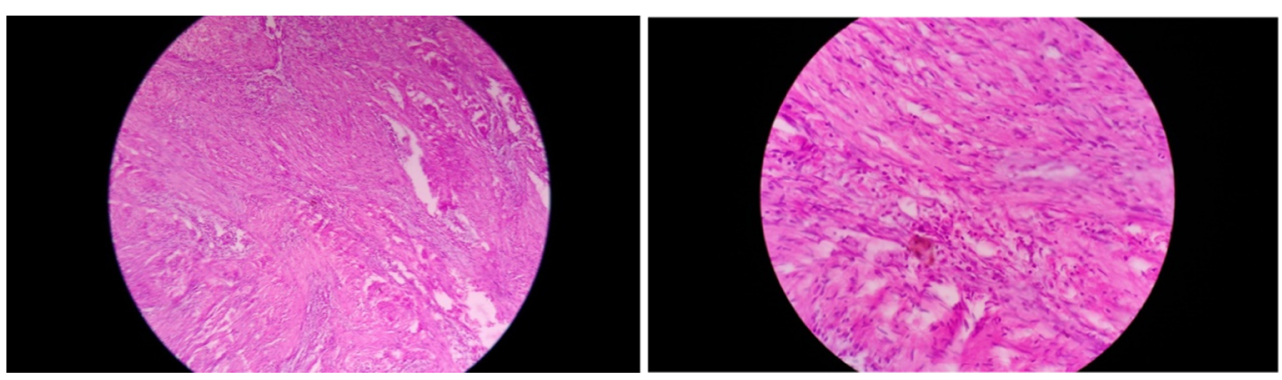


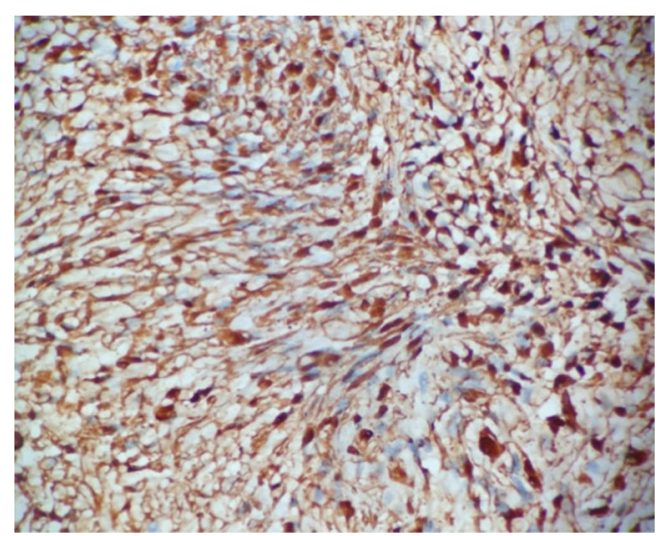

Fig. 4 S-100 protein diffuse positivity seen in IHC

uniformly S-100 positive (Fig. 4). These findings were consistent with benign schwannoma.

\section{Discussion}

Schwannomas are benign tumors arising from Schwann cells of peripheral, motor, sympathetic or cranial nerves. They are commonly found in head and neck, upper and lower extremities, and less commonly gastrointestinal tract and in retroperitoneum. The description of schwannomas was done first by Verocay in 1908 and in 1920 Antoni subclassified into two distinct histologic patterns.

Adrenal schwannoma is a rare type of adrenal incidentaloma with fewer than 20 cases reported in the literature. Adrenal medulla is innervated by the phrenic nerve, vagus nerve, and sympathetic trunk. Adrenal schwannoma arises from Schwann cells of these nerves $[1,2]$. On computed tomography schwannoma appears as a well-circumscribed homogenous masses and sometimes as heterogeneous masses due to cystic degeneration. MRI findings are usually nonspecific.

The differential diagnosis of adrenal schwannoma includes adrenal adenoma, adrenal metastasis, leiomyoma, neuroblastoma, ganglioneuroma, ganglioneuroblastoma, pheochromocytomas, and solitary fibrous tumors [4-6].

It is very difficult to diagnose adrenal schwannoma preoperatively. Surgical resection and histological examination of the operative specimen establishes diagnosis of adrenal schwannoma.

Histological features of adrenal schwannomas are like those of schwannomas found in other sites.

Schwannomas can be categorized as either conventional or cellular schwannomas based on their histologic features.
Conventional schwannomas consist of spindle-shaped cells with alternating compact hypercellular areas and loosely textured hypocellular areas, termed Antoni A and Antoni B respectively. Verocay bodies and thick-walled hyalinized blood vessels can also be seen $[1,7]$. Cellular schwannomas consist only Antoni A areas with intersecting fascicles of spindle cells and are devoid of hypocellular Antoni B areas and Verocay bodies with increased mitotic activity [2, 8]. Immunohistological staining is essential for diagnosis, as tumor cells are positive for S-100 protein and vimentin.

\section{Conclusion}

We report a case of an adrenal schwannoma incidentally discovered in a 68-year-old male patient during work-up for dysuria. Adrenal schwannomas are extremely rare tumors and preoperatively they are difficult to diagnose. Definitive diagnosis of schwannoma can only be possible by surgical excision and histopathological and immunohistochemical evaluation of the operative specimen.

\section{References}

1. Korets R, Berkenblit R, Ghavamian R (2007) Incidentally discovered adrenal schwannoma. JSLS 11(1):113-115

2. Lau SK, Spagnolo DV, Weiss LM (2006) Schwannoma of the adrenal gland: report of two cases. Am J Surg Pathol 30(5):630-634

3. Arena V, De Giorgio F, Drapeau CMJ, Monego G, De Mercurio D, Capelli A (2004) Adrenal Schwannoma. Report of two cases. Folia Neuropathol 42(3):177-179

4. Thompson LD (2002) Pheochromocytoma of the Adrenal Gland Scaled Score (PASS) to separate benign from malignant neoplasms: a clinicopathologic and immunophenotypic study of 100 cases. Am J Surg Pathol 26(5):551-566

5. Bongiovanni M, Viberti L, Giraudo G et al (2000) Solitary fibrous tumour of the adrenal gland associated with pregnancy. Virchows Arch 437:445-449

6. Nishida S, Tanimura A, Takasaki S et al (1995) Surgically resected adrenal leiomyoma: report of a cases. Surg Today 25:455-457

7. Suzuki K, Nalanish A, Kuurosaki Y, Nogaki J, Takaba E (2007) Adrenal schwannoma: CT and MRI findings. Radiat Med 25(6): 299-302

8. Hsiao HL, Li CC, Lin HC, Yeh HC, Huang CH, Wu WJ (2008) Adrenal schwannoma treated with laparoscopic adrenalectomy: a case report. Kaohsiung J Med Sci 24(10):553-557 